Please send trade news information and illustrations to Arveen Bajaj at the $B D J$, Nature Publishing Group, The Macmillan Building, 4-6 Crinan Street, London N1 9XW.

Trade news is provided as a service to readers using text and images from the manufacturer, supplier or distributor and does not imply endorsement by the $B D J$. Normal and prudent research should be exercised before purchase or use of any product mentioned.

\section{New microhybrid composite restorative}

New Diamond Finish from Kemdent is an advanced, easy to use, microhybrid composite available in packable syringes and flowable application caps. In addition the company has used the latest nano-technology bonding system in the new Diamond Bond light-curing, selfetching adhesive which enables dentists to combine the advantages of Diamond Carve GIC (glass ionomer cement) with the aesthetic benefits offered by the Diamond Finish composite technology in a single procedure. This saves valuable chair time and produces superior restoration outcomes.

The unique nano-reinforced, self-etching bond system is specially designed to bond Diamond Carve GIC to Diamond Finish composites. It complies to a specific bond strength for 'sandwich' construction restorations. Diamond Finish Packable is designed for Class I to $\mathrm{V}$ restorations and is available in $5 \times 4 \mathrm{~g}$ syringes and refills of $2 \times 4 \mathrm{~g}$ in shades A2, A3, A3.5, B2, B3, C2 and 0A2. Offer prices are $£ 71.25$ pack, with refills at £29.96. Diamond Finish Flowable is designed for Class II, IV and V restorations and is available in $25 \times 0.25 \mathrm{~g}$ caps in shades A2, A3 and A3.5 with an offer price of $£ 29.96$. Diamond Bond light-curing, self-etching adhesive comes in $2 \times 4 \mathrm{ml}$ and the offer price is $£ 41.25$. Orders before 30 June can take advantage of a $25 \%$ introductory discount.

Reader response number 50

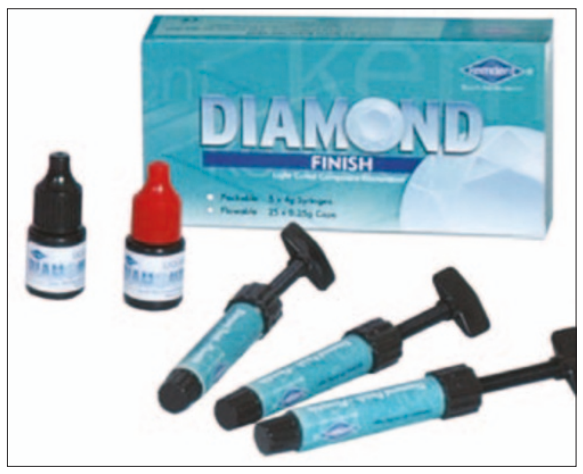

\section{New generation dental system}

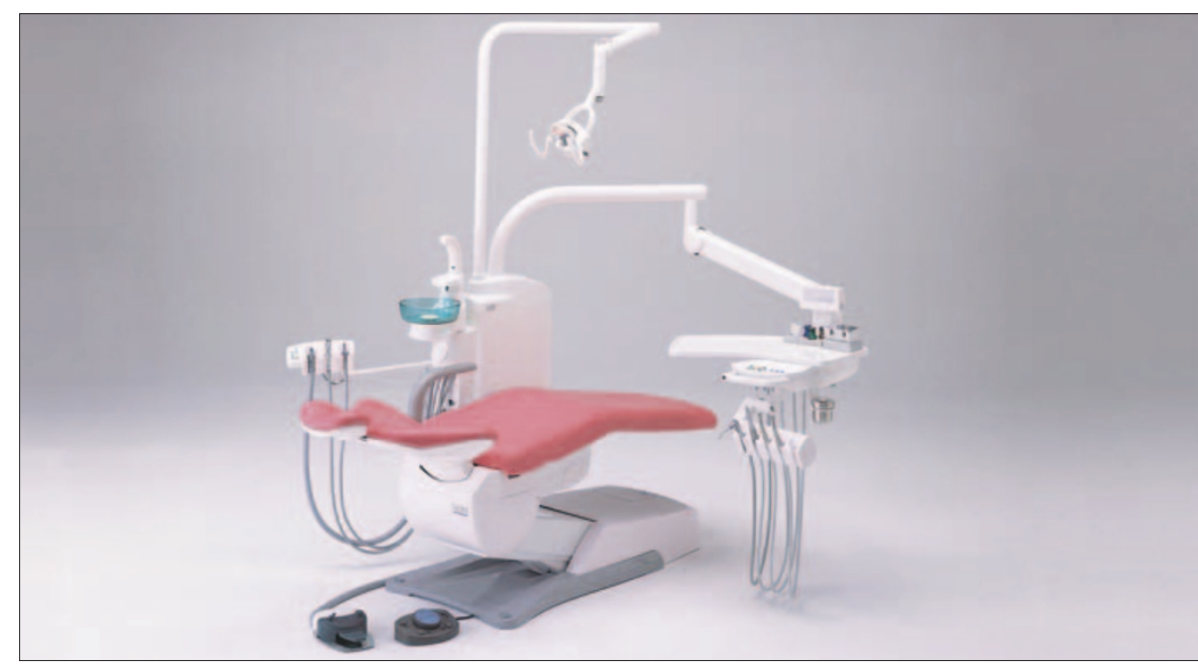

Takara Belmont has recently introduced the Clesta II dental system. Operators can create their ideal dental system as the Clesta II is totally flexible and comes in four configurations. Each configuration can also be ordered in 'Air' and 'Electric' mode for increased choice.

The electric E-type offers high specification and technical uplift with fibre-optics, micro motor and digital display all as standard. Every configuration can be ordered in left or right hand format from the UK factory.

The Clesta II comes with a cuspidor that rotates through $180^{\circ}$ to allow the rinsing bowl to be positioned over the patient or out of the way for clear access for the dental nurse. It also comes with an easy to remove detachable glass bowl to improve hygiene and speed of cleaning.

The lightweight, over-the-patient swing arm is engineered to provide precision positioning. Each chair comes with a Belmont 720 dental light that incorporates a composite safe mode as standard to increase working time.

Finally, each panel has a perfect fit so there are no gaps to harbour bacteria. The under-chair service centre integrates all umbilical hoses into the low line base and the cuspidor is plumbed through the chair base to minimise visible tubing.

Reader response number 51

\section{Improved visibility and accessibility}

The Star Dental range of handpieces, airmotors and scalers available from DentalEZ comprise the Solara and 430 Series handpieces. These incorporate the unique Purple Power Lube Free turbine which uses ceramic ball bearings. This increases the bearing life and eliminates the need for lubrication, thus no residual oil is expelled into the oral cavity to jeopardise adhesive techniques.

All Star handpieces have midi compact head for improved visibility and accessibility to the oral cavity and high torque for increased efficiency. The new Solara fibre optic handpiece has lightweight titanium construction which reduces hand and wrist fatigue while the Starflex handpiece uses steel bearings. Reader response number 52 


\section{IMPRESSION MATERIALS}

\section{Sterile silicone}

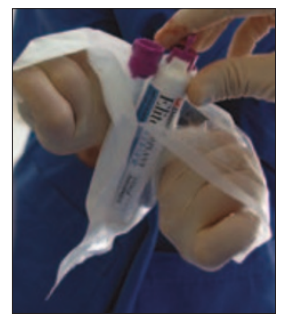

Zhermack have launched a unique sterile 'A' silicone for the immediate loading implant procedure where the impression is taken immediately after surgery. This will reduce the risk of post-operative complications as the product is also biocompatible to avoid tissue damage if a fragment remains in the mucosa. It also offers the additional security of being radiopaque.

The Elite Implant comes in three viscosities, heavy $(50 \mathrm{ml})$ which is used on its own for edentulous patients and is very rigid, medium (50ml) which is used on its own for partially dentate patients or with light $(5 \mathrm{ml})$ for use around the implants and preparations.

Reader response number 53

\section{Uniform snap-set}

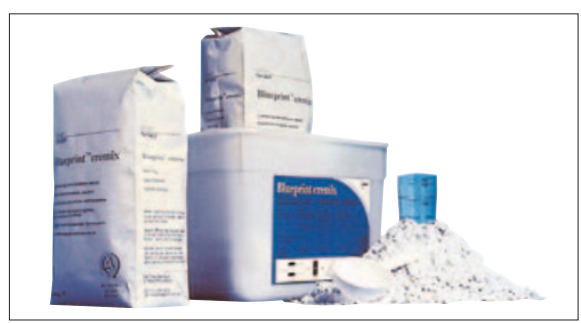

Blueprint Cremix is a lead-free, easy mixing alginate that is suitable for full and partial oral impressions, including those for orthodontics, prosthetics, occlusion analyses and study models.

The light, wettable, powder results in a creamy consistency and its dust-free nature ensures no eye irritation or powder inhalation when mixing. The resulting impressions have high precision (due to high strength and tear resistance) as well as offering ample working time with a short setting time. The snap-set ensures a uniform set throughout the impression and reduces the risk of distortion during setting.

Reader response number 54

New for mixing machines

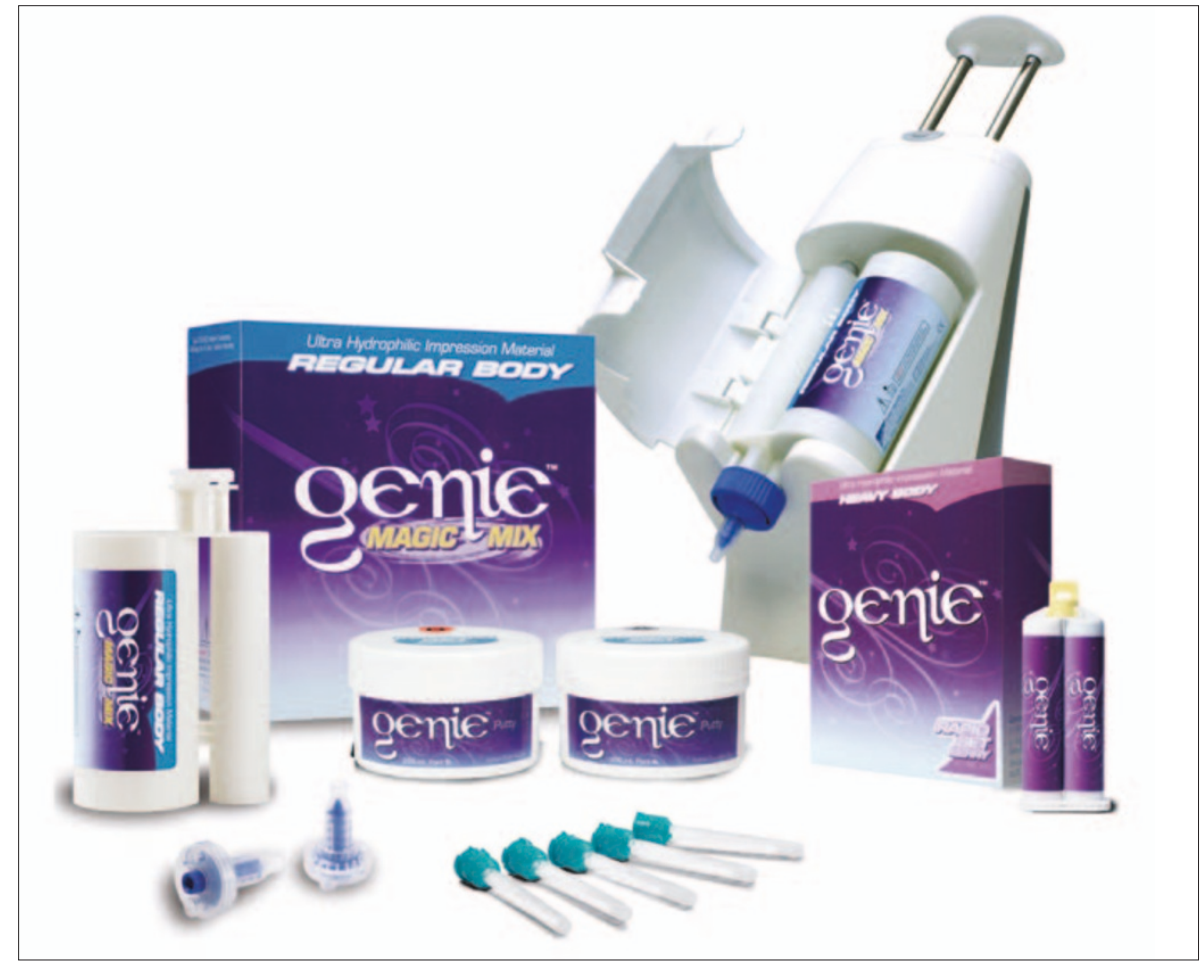

Sultan Chemists has announced the launch of the Genie Magic Mix exclusively for impression material mixing machines. A new addition to its Genie line of ultra hydrophilic vinyl polysiloxane dental impression materials, it is available directly through Sultan's global network of distribution partners. It comes in a 380ml economy size, delivering 5\% more material than foil bag systems through its new cartridge style. Convenient and easy to use, there is no activation required -

\section{Greater flexibility}

The Dental Directory offers practices a choice of two types of denture reline kits (hard and soft) from UnoDent for greater flexibility. The self-curing, denture rebase acrylic is formulated to set quickly making it ideal for chairside use. Its formulation is non-irritating for the patient and is de-lamination proof for greater durability. If well looked after, the soft formulation will remain soft and cush- just load and go and there is a choice of two set times, rapid $=2: 10$ and standard $=4: 30$.

Genie is ultra hydrophilic, providing a solution that is extremely reliable and forgiving in any clinical oral environment, and removing any possibility of moisture compromising results. All Genie viscosities flow extremely well under pressure, ensuring the best possible coverage of all surfaces of prepared teeth.

Reader response number 55

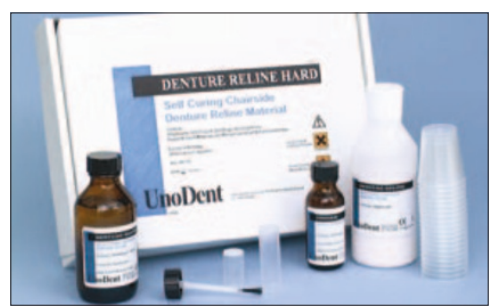

ion-like for over a year. Each kit consists of $100 \mathrm{~g}$ of powder, $100 \mathrm{ml}$ of liquid, $30 \mathrm{ml}$ of primer and some accessories.

Reader response number 56 


\section{Accurate results}

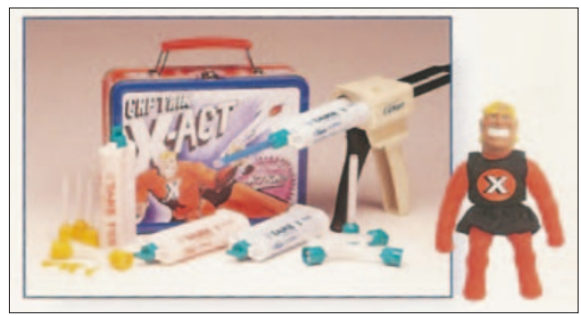

Kerr's Take 1 builds on the heritage of Extrude, its first family of impression materials, employing advances made in vinyl material, filler technology and hydrophilic monomers. Take 1 is actually a blend of five vinyls and a bimodal filler system. In addition to Take 1's surfactant, Kerr also included a patented hydrophilic additive. This unique, proprietary formulation results in a material that works consistently and easily in virtually any oral environment.

The optimal physical properties of the product allows improved elongation and tear strengths, which enhance marginal integrity and enables you to take the most precise impressions even in a wet environment. The patented hydrophilic additive provides excellent surface energy, resulting in detailed reproduction even in the presence of blood and saliva. Whilst being accurate, Take 1 is easy to remove from the mouth to minimise patient discomfort and also reduces the risks associated with periodontically compromised teeth as well as implants.

The bright, contrasting colours provide easy to read impressions and it is available in five viscosities and two setting times.

Reader response number 57

\section{High elasticity alginate}

Kromopan alginates from Astek Innovations feature smooth, creamy mixing, a pleasant light mint flavour, high elasticity on final set and 100 hours dimensional stability rating. Kromopan can be used for impressions being taken for prosthetics (including chromes), orthodontics or simply for antagonist work, and can also be multi-poured.

As part of Astek Innovations summer launch with

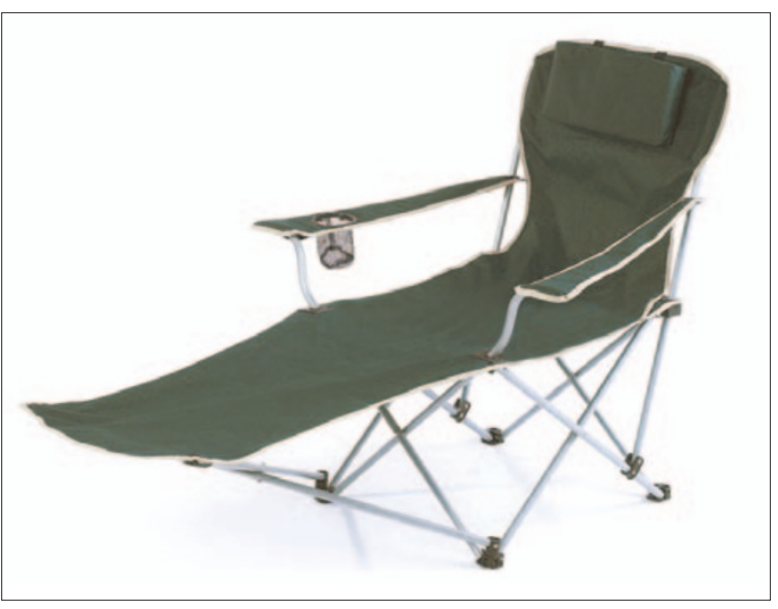
Kromopan, each economy pack (20 x 450 gm bags) comes with a free 'Malibu' reclining chair complete with drinks holder and head rest pillow. The 'Malibu’ would generally cost up to £40. Dentists wishing to initially evaluate Kromopan with a smaller purchase can take advantage of a special low priced introductory kit containing 450gm bag, tub and measures for £5.95 saving $30 \%$ off the list price and then graduating up to the 'Malibu' offer later. Offer ends 30 June and is subject to availability.

Reader response number 58

\section{Faster impressions}

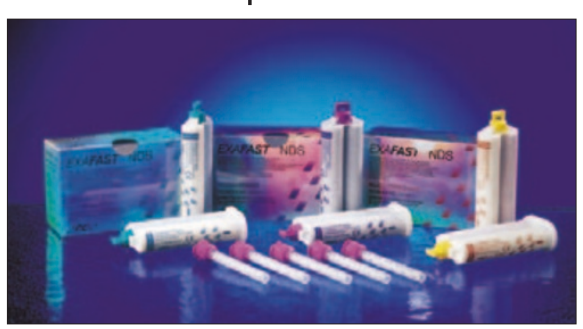

GC has produced a range of impression materials that set in super fast time. The Exafast NDS family of addition cured silicones provide high quality, accurate and consistent impressions. The product allows you to produce very smooth and highly precise impressions in only two minutes, thus improving productivity and maximising the comfort of your patients.

GC Exafast Putty is an extremely fast setting putty material, ideally for use in the single step technique. In combination with other GC Exafast NDS materials its working time of 45 seconds and setting time of 2 minutes 15 seconds offers a combination of speed and precision.

The Exafast wash material is available in three viscosities to suit your preferred technique. All are supplied in cartridges for speed and ease of application. As the material sets so quickly there is less risk of distortion due to movement during the setting. Reader response number 59 